\title{
Insufficient evidence for photodynamic therapy use in periodontitis
}

\author{
Abstracted from \\ Azarpazhooh A, Shah PS, Tenenbaum HC, Goldberg MB. \\ The effect of photodynamic therapy for periodontitis: A Systematic Review and Meta-Analysis. \\ J Periodontol 2010; 81: 4-14. \\ Address for correspondence: Community Dental Health Services Research Unit and Division of Endodontics, \\ University of Toronto, 124 Edward St., Toronto, ON M5G 1G6, Canada. \\ E-mail: amir.azarpazhooh@dentistry.utoronto.ca
}

\section{Question: Does photodynamic therapy (PDT) improve periodontal outcomes compared to scaling and root planing (SRP) or no treatment}

Data sources The Cochrane Central Register of Controlled Trials, MEDLINE, EMBASE, CINAHL, Health and Psychosocial Instruments, HealthSTAR (OVID), Allied and Complementary Medicine and the International Pharmaceutical Abstracts. Abstracts from 2007 to 2009 of the annual meetings of the American Academy of Periodontology, International Association of Dental Research and the American Association of Dental Research.

Study selection Randomised and quasi-randomised studies reported in any language comparing PDT as a primary or adjunctive therapy to no treatment, placebo or scaling and root planing (SRP). Eligible studies were those that included participants over 18 years of age and who had periodontitis, and where the primary outcome measurement was clinical attachment loss (CAL) and changes in probing depth.

Data extraction and synthesis Two reviewers reviewed, assessed and rated study quality and extracted relevant data. It is not reported how these data were collated. The quality of included studies was assessed according to Cochrane risk of bias domains. Mean difference (MD) and $95 \%$ confidence intervals $(\mathrm{Cl})$ were extracted. Data were combined in a metaanalysis where possible using the random-effects model. Homogeneity was assessed using the Cochrane test and heterogeneity assessed using $l^{2}$. Results Five studies at moderate to high risk of bias were included. The studies differed markedly in design and were clinically heterogenous. Studies that compared PDT to no treatment found no difference in CAL whereas those that compared PDT plus SRP $(n=26)$ to those receiving just SRP ( $n=26)$ gave a MD of $0.34 \mathrm{~mm}$ with $95 \% \mathrm{Cl} 0.05$ to 0.63 $\mathrm{mm}$. Three studies that compared PDT alone to SRP alone showed a reduction in probing depth in favour of SRP (MD - $0.21,95 \% \mathrm{Cl}-0.40$ 0.02). In three studies that compared PDT plus SRP to SRP alone the $\mathrm{MD}$ was $0.25 \mathrm{~mm}$ ( $95 \% \mathrm{Cl}: 0.04$ to $0.45 \mathrm{~mm}$ ).

Conclusions There is insufficient evidence that photodynamic therapy as an independent treatment or as an adjunct to scaling and root planning is superior to SRP alone.

\section{Commentary}

Subgingival debridement is the most common method to treat periodontitis, and different adjunctive therapies have been proposed in order to improve clinical and/or microbiological outcomes. Photodynamic therapy (PDT) has been proposed as one of these adjunctive therapies, since the activation of dyes by light will lead to the formation of singlet oxygen species, which are cytotoxic.

This systematic review compares PDT, both as sole or as adjunctive therapy, with no treatment, placebo or mechanical therapy alone (SRP). For the screening of papers, no hand searching was conducted. After the selection process, nine papers were initially included, but only five of them were suitable for meta-analysis. The exclusion of the other four papers from the systematic review may not be adequate.

The heterogeneity of the selected papers is clearly described, including type of disease, study duration (for 3 weeks to 6 months), or designs (including split mouth). The risk of bias was considered as medium to high. In addition, all studies had a limited sample size (from 10 to 33 patients). Also, the PTD protocols showed differences with regards to the photosensitiser use or the selected laser (including wave length, power and irradiation time).

Some meta-analyses (with two or three studies) were conducted, comparing SRP plus PTD versus SRP and PTD versus SRP. One study was not included in the meta-analyses (Braun et al. 2008), and according to the criteria defined by the authors, it should have been excluded.

Overall, the differences were of small magnitude and tended to favour SRP when compared with PDT (significant for pocket depth changes), and the combination of SRP plus PDT when compared with SRP alone (significant for attachment level and pocket depth changes). Adverse effects and patient-based variables could not be assessed due to the lack of information in the selected papers.

In the discussion the limitations of this systematic review are acknowledged, although the authors tended to focus on the limitations of periodontal probing. The interest of the industry in the topic and the issue of the sponsored research, which is mentioned in the introduction, were not further discussed.

- Adequately powered and designed studies are needed to assess PDT therapy in the basic therapy phase of periodontitis, as an adjunct to subgingival debridement

- The available evidence to support the use of PTD in periodontitis therapy can only be considered as preliminary.

David Herrera Section of Periodontics, Faculty of Odontology, University Complutense, Madrid, Spain

1. Braun A, Dehn C, Krause F, Jepsen S. Short-term clinical effects of adjunctive antimicrobial photodynamic therapy in periodontal treatment: a randomized clinical trial. / Clin Periodontol 2008; 35: 877-884.

Evidence-Based Dentistry (2011) 12, 46. doi:10.1038/sj.ebd.6400791 